\title{
El tratamiento de la hipertensión arterial a la hora de acostarse mejora la reducción del riesgo cardiovascular
}

Treatment of hypertension at bedtime, improves cardiovascular risk reduction

Hermida, R. C., et al. European Heart Journal;2019(0):1-12. doi:10.1093/eurheartj/ehz754

\section{Comentado de:}

Hermida, R. C., et al. European Heart Journal;2019(0):1-12. doi:10.1093/eurheartj/ehz $754^{1}$.

\section{Objetivos}

El Ensayo de Cronoterapia Hygia, realizado dentro del entorno clínico de atención primaria, fue diseñado para evaluar si la terapia antihipertensiva a la hora de acostarse en comparación con lo habitual al despertar, ejerce una mejor reducción de riesgo de enfermedad cardiovascular (ECV).

\section{Métodos y resultados}

En este estudio multicéntrico, controlado y prospectivo, se incluyeron 19.084 pacientes hipertensos (10.614 hombres), de $60,5 \pm 13.7$ años de edad para ingerir la dosis diaria completa de $\geq 1$ medicamentos para la hipertensión, en una relación 1:1, a la hora de acostarse $(n=9.552)$ o al despertar $(n=9.532)$.

En la inclusión y en cada visita clínica programada (al menos una al año) durante todo el seguimiento, se realizó un monitoreo ambulatorio de la presión arterial (MAPA) durante 48 hs. La media de seguimiento fue 6,3 años, durante el cual 1.752 pacientes experimentaron el resultado primario compuesto de ECV (muerte por ECV, infarto de miocardio, revascularización coronaria, insuficiencia cardíaca o accidente cerebrovascular).

Los pacientes del grupo toma a la hora de acostarse mostraron, en comparación con el régimen de toma al despertar, una relación de riesgo significativamente menor, ajustada por características influyentes significativas de edad, sexo, diabetes tipo 2, enfermedad renal crónica, tabaquismo, colesterol, tabaquismo, presión arterial (PA) media sistólica durante el sueño, disminución relativa de la PA sistólica en el tiempo de sueño y evento ECV previo. Esto fue cierto tanto para el resultado primario compuesto de ECV (HR 0,55; IC del $95 \% 0,50$ a 0,61; $P<0,001$ ), como para cada uno de sus componentes individuales $(P<0,001$ en todos los casos), es decir, muerte por ECV $(0,44 ; 0,34$ a 0,56$)$, infarto de miocardio $(0,66 ; 0,52$ a 0,84$)$, revascularización coronaria $(0,60 ; 0,47$ a 0,75$)$, insuficiencia cardíaca $(0,58 ; 0,49$ a 0,70$)$ y accidente cerebrovascular $(0,51 ; 0,41$ a 0,63$)$.

\section{Conclusiones}

La ingestión de rutina por parte de pacientes hipertensos de $\geq 1$ medicamentos recetados para reducir la presión sanguínea a la hora de acostarse, en lugar de al despertar, da como resultado un mejor control de la PA (disminución significativamente mejorada de la PA durante el sueño y una disminución relativa de la PA relativa al tiempo de sueño, es decir, caída de la PA) y, más importante, disminución marcada de la ocurrencia de eventos cardiovasculares.

Conflicto de interés de los autores: : El Proyecto Hygia es una red de investigación independiente, respaldada por subvenciones sin restricciones.

\section{Comentario}

Si bien estudios previos ${ }^{2-5}$ ya mostraban un efecto beneficioso de la dosificación nocturna de la terapia antihipertensiva, eran necesarios estudios multicéntricos para probar esta estrategia.

El Hygia Chronotherapy Trial es el primer estudio multicéntrico, basado en MAPA, con una duración significativa en el seguimiento y realizado dentro del entorno médico de atención primaria que evaluó una gran cohorte prospectiva de pacientes hipertensos, buscando confirmar la hipótesis de si la ingesta de la dosis diaria completa de $\geq 1$ medicamentos para reducir la presión sanguínea a la hora de acostarse, mejora el control de presión arterial y ofrece una mejor protección contra los principales ECV.

Los resultados del estudio muestran, que los pacientes que tomaban la medicación al acostarse tenían una reducción, de casi la mitad de riesgo de sufrir o morir de un infarto de miocardio, accidente cerebrovascular, insuficiencia cardíaca o de requerir un procedimiento de revascularización coronaria, en comparación con quienes la tomaban al despertar.

Este estudio también reveló que la presión arterial tomada poco antes de ir a dormir es el indicador independiente más importante de riesgo de padecer algún problema cardiovascular.

La principal limitación del estudios de cronoterapia Hygia es, que los hallazgos requieren validación y extrapolación a otros grupos étnicos.

Además, el ensayo no asignó a los participantes a clases específicas de medicamentos para la hipertensión, más bien, el tratamiento fue elegido por cada médico respetando la práctica clínica actual. Esto resultó en un desequilibrio en el número de pacientes por clase de medicación. No obstante, las diferencias en las tasas de prescripción entre las diferentes clases de medicamentos reflejan las preferencias terapéuticas en el entorno de atención primaria, siendo también el diseño del estudio más cercano a la práctica clínica habitual, que puede a su vez, ser una de las principales ventajas.

\section{Conclusiones de la comentadora}

Teniendo en cuenta que las actuales directrices (guías) para el tratamiento de la hipertensión no mencionan o recomiendan ninguna hora preferida para la toma de la medicación y que la toma durante la mañana ha sido la recomendación más común por parte de los médicos (basada en el objetivo de reducir los niveles matutinos de presión arterial), el estudio aquí comentado nos brinda un resultado interesante a tener en cuenta, ya que sugiere que se puede reducir en más de un $50 \%$ los problemas cardiovasculares en pacientes con hipertensión, sin ningún costo adicional, aplicando la simple estrategia de administrar la medicación al irse a dormir en vez de al despertarse. 
Yanina Avendaño [ Servicio de Medicina Familiar y Comunitaria, Hospital Italiano de Buenos Aires, Argentina. yanina.avendano@hospitalitaliano.org.ar ]

Avendaño, Y. El tratamiento de la hipertensión arterial a la hora de acostarse mejora la reducción del riesgo cardiovascular. Evid Actual Pract Ambul. 2020;22(04):e002030. Comentado de: Hermida, R.C. Bedtime hypertension treatment improves cardiovascular risk reduction: the Hygia Chronotherapy Trial. European Heart Journal;2019(0):1-12. doi:10.1093/eurheartj/ehz754. PMID: 31641769.

\section{Referencias}

1. Hermida RC, Crespo JJ, Domínguez-Sardiña M, Otero A, Moyá A, Ríos MT, et al. Bedtime hypertension treatment improves cardiovascular risk reduction: the Hygia Chronotherapy Trial. European Heart Journal. 2019 oct;Available from: 10.1093/eurheartj/ehz754

2. Hermida RC, Ayala DE, Mojón A, Fernández JR. Influence of circadian time of hypertension treatment on cardiovascular risk: Results of the MAPEC study. Chronobiology International. 2010;27(8):1629-1651. Available from: 10.3109/07420528.2010.510230.

3. Sobiczewski W, Wirtwein M, GruchaM, Kocić I. Mortality in hypertensive patients with coronary heart disease depends on chronopharmacotherapy and dipping status. Pharmacological Reports. 2014;66(3):448-452. Available from: 10.1016/j.pharep.2013.12.009.

4. Fujiwara T, Hoshide S, Yano Y, Kanegae H, Kario K. Comparison of morning vs bedtime administration of the combination of valsartan/amlodipine on nocturnal brachial and central blood pressure in patients with hypertension. Journal of Clinical Hypertension. 2017;19(12):1319-1326. Available from: 10.1111/jch.13128.

5. Roush GC, Fapohunda J, Kostis JB. Evening Dosing of Antihypertensive Therapy to Reduce Cardiovascular Events: A Third Type of Evidence Based on a Systematic Review and Meta-Analysis of Randomized Trials. Journal of Clinical Hypertension. 2014;16(8):561-568. Available from: $10.1111 /$ jch.12354. 